\title{
TGFBI gene mutations analysis in Chinese families with corneal dystrophies
}

\author{
XIAOJUAN WANG ${ }^{1}$, MING YING $^{2}, \mathrm{CHANGBO} \mathrm{FU}^{1}, \mathrm{YUCHUAN} \mathrm{WANG}^{2}$ and NINGDONG $\mathrm{LI}^{2}$ \\ ${ }^{1}$ Xuzhou Eye Institute, The First People's Hospital of Xuzhou, Xuzhou, Jiangsu 221000; ${ }^{2}$ Tianjin Eye Hospital, \\ Tianjin Eye Institute, Tianjin Key Lab of Ophthalmology and Visual Science, Tianjin 300020, P.R. China
}

Received February 4, 2016; Accepted January 26, 2017

DOI: $10.3892 / \mathrm{mmr} .2017 .6414$

\begin{abstract}
The aim of the present study was to examine the clinical features of three Chinese families with autosomal dominant corneal dystrophy (CD) and examine transforming growth factor- $\beta$-induced $(T G F B I)$ gene mutations in these families. The TGFBI gene mutations were detected using direct sequencing of the whole coding regions and exon-intron boundaries of the TGFBI gene in the affected members from the three families with $\mathrm{CD}$. The phenotypes of all affected individuals in the three families were observed via slit lamp examination. Sections of the cornea were used for biopsy following keratoplasty. Three types of TGFBI gene mutations, R124C, H626R and R124H, were detected in the patients from these three families. One family, with the R124C mutation, was diagnosed with lattice corneal dystrophy type 1, and the family with the H626R mutation was diagnosed with lattice corneal dystrophy type IIIB. The family with the R124H mutation was diagnosed with granular corneal dystrophy type 2 . The TGFBI gene mutations were considered underlying factors in the molecular mechanism underlying the pathogenesis of cornea dystrophy. Therefore, the detection of TGFBI gene mutations may be useful in the differential diagnosis of CD.
\end{abstract}

\section{Introduction}

Corneal dystrophies (CDs) are a group of heterogeneous inherited diseases with non-inflammatory, bilaterally progressive corneal opacities, which often lead to recurrent corneal erosion and visual impairment. According to the affected layer in the cornea, CDs can be categorized as epithelial, subepithelial, Bowman's layer, stromal or endothelial using slit-lamp imaging, confocal microscopy

Correspondence to: Dr Ningdong Li, Tianjin Eye Hospital, Tianjin Eye Institute, Tianjin Key Lab of Ophthalmology and Visual Science, 4 Gansu Road, Heping, Tianjin 300020, P.R. China E-mail: ningdongli2015@sina.com

Key words: corneal dystrophy, transforming growth factor- $\beta$-induced, mutation or histological staining However, it has been found that the clinical classifications of CDs based entirely on ophthalmological and histopathologic examinations are limited in scope and effectiveness (1).

Advances in molecular genetics have mapped CDs to eleven chromosomes: 1, 2, 5, 9, 10, 12, 13, 16, 17, 20 and $\mathrm{X}(2)$. Several genes, including the transforming growth factor $\beta$-induced (TGFBI) gene (OMIM 601692), carbohydrate sulfotransferase 6 gene, gelsolin gene, keratin 3 gene, keratin 12 gene and surface marker 1 gene, have so far been identified as responsible for CDs $(3,4)$. Among these, TGFBI-associated CDs have provided the most reliable evidence. To date, at least five autosomal dominant CDs have been confirmed to be associated with mutations in the TFGBI gene: i) Reis-Bücklers CD (RBCD), ii) lattice CD type 1 (LCDI), iii) Thiel-Behnke CD, iv) granular CD type 1 (GCD1) and v) GCD2 $(5,6)$.

As $C D$ has significant genetic heterogeneity, different TGFBI gene mutations can lead to the same phenotype, for example, R124C, V625D, V505D, H626R and other mutations are associated with LCDI (7-9); whereas the same TGFBI mutation in a different ethnic population can exhibit different clinical phenotypes, for example, the R124C mutation is responsible for GCD2, LCDI and RBCD (10-12). Due to the complexity of phenotypic and genetic heterogeneity, the ability of ophthalmologists and geneticists to correctly diagnose and classify CDs is a challenge, as is understanding their phenotype-genotype aspects. In the present study, three Chinese families affected by different types of CD were recruited, and the gene mutations responsible for the disease were identified. The results aimed to provide an insight into the clinical-molecular correlations of the disease.

\section{Materials and methods}

Patients and subjects. In the present study, three Chinese pedigrees comprising individuals diagnosed with LCDI, LCD IIIB and GCD2, respectively, were recruited from the Tianjin Eye Hospital (Tianjin, China) between August 2013 and July 2014. The study was approved by the Ethics Committee of Tianjin Eye Hospital. Informed, written consent was obtained from each of the participants in accordance with the Declaration of Helsinki prior to the collection of their peripheral blood. The participating members underwent careful ophthalmic examination, including vision tests, slit-lamp biomicro-examination 
and anterior segment imaging. In addition, 100 unrelated subjects without CD were recruited from the Tianjin Eye Hospital as normal controls for the present study.

Genetic analysis. Genomic DNA was extracted from the peripheral blood samples using a genomic DNA miniprep kit for blood (Roche Diagnostics, Indianapolis, IN, USA). DNA integrity was evaluated using $1 \%$ agarose gel electrophoresis. The DNA fragments, which encoded regions of the TGFBI gene, were amplified using polymerase chain reaction (PCR). The sequences of the primers were designed according to a previous study by Stix et al (13) (Table I). Standard PCR reactions were preformed in a $50 \mu \mathrm{l}$ reaction mixture including $1 \mu \mathrm{l}$ of 10X PCR buffer, $50 \mathrm{ng}$ template DNA, $0.1 \mu \mathrm{M}$ of each of the forward and reverse primers, $300 \mu \mathrm{M} \mathrm{dNTP,} 5 \mathrm{mM} \mathrm{MgCl}_{2}$ and $0.3 \mathrm{U}$ Hotstart Taq. The PCR program used for DNA amplification was as follows: $95^{\circ} \mathrm{C}$ for $3 \mathrm{~min}$; followed by 20 cycles at $95^{\circ} \mathrm{C}$ for $30 \mathrm{sec}, 53-64^{\circ} \mathrm{C}$ for $30 \mathrm{sec}$, and $72^{\circ} \mathrm{C}$ for $45 \mathrm{sec}$; additional 20 cycles at $95^{\circ} \mathrm{C}$ for $30 \mathrm{sec}, 55^{\circ} \mathrm{C}$ for $30 \mathrm{sec}, 72^{\circ} \mathrm{C}$ for $30 \mathrm{sec}$; and a final extension at $72^{\circ} \mathrm{C}$ for $7 \mathrm{~min}$, using the Gene Amp PCR 9700 system (Applied Biosystems; Thermo Fisher Scientific, Inc., Waltham, MA, USA).

Each PCR fragment was purified with a TIANgel Midi DNA Purification kit (Tiangen Biltech Co., Ltd, Beijing, China), and the two strands were subsequently sequenced on an ABI 3130 automated DNA sequencer (Applied Biosystems; Thermo Fisher Scientific, Inc.). The sequence results were compared with the wild-type TGFBI sequence (GenBank NG_012646.1; https://www.ncbi.nlm.nih.gov/nuccore/NG_012646.1). The other family members and 100 Chinese control individuals were also screened for the presence of TGFBI mutations by PCR amplification and direct sequencing of PCR products.

Histopathological examination. The corneal specimens obtained using penetrating keratoplasty were processed for examination using light microscopy. The tissues were fixed in $10 \%$ formalin and embedded in paraffin. Cross sections of each button were prepared by free-hand sectioning at a thickness of $\sim 10 \mathrm{~mm}$ and were stained with Congo red.

\section{Results}

Family with LCDI. In the first family, comprising three generations, four affected members and five unaffected members were examined (Fig. 1A). The symptoms of all patients began with episodes of acute ocular pain, redness and photophobia at 20 years of age. Slit-lamp examination revealed dots and fine lattice lines in the anterior corneal stroma (Fig. 1B). The small lattice-shaped opacity is a typical clinical feature LCDI, which can be observed using direct and retroillumination. The mutation analysis showed that a heterozygous c.371 C>T mutation (R124C) in exon 4 of TGFBI was present in the affected members, but not in the unaffected members or controls (Fig. 1C).

Family with LCDIIIB. In the second three-generation family, six affected members and eight unaffected members were examined (Fig. 2A). Thick and ropy lattice lines of amyloid were visible under direct illumination of the corneas of the proband patient II-1 (Fig. 2B). This patient was referred to hospital with visual acuity loss to 0.2 and underwent perforating keratoplasty on her right eye at 56 years old. The corneal specimens obtained during surgery were submitted for histologic examination in the present study, which revealed large patchy amyloid deposits in the subepithelial layer and deeper corneal stroma (Fig. 2C). The clinical symptoms of the other affected family members were similar to those of the proband. Mutation analysis showed that a heterozygous c.1877 $\mathrm{A}>\mathrm{G}$ mutation (H626R) in exon 14 of TGFBI was present in the proband and other affected members, but not in the unaffected members or 100 controls (Fig. 2D).

Family with GCD2. In this three-generation family, five affected patients and seven unaffected members were examined (Fig. 3A). The proband (a 22-year-old female patient; III:2) had recurrent corneal epithelial erosion with visual impairment, and symptoms of redness, photophobia, lacrimation and foreign-body sensation in both eyes. The visual acuity recovered to 1.0 when corneal epithelial erosion disappeared. Slit-lamp examination showed bilateral scattered grayish small dot, annular and snow flake-like opacities in the superficial mid-stroma of the central cornea (Fig. 3B). Mutation analysis showed that a heterozygous c.371 G>A mutation $(\mathrm{R} 124 \mathrm{H})$ in exon 4 of $T G F B I$ was identified in the proband and other affected members, but not in the unaffected members and healthy controls (Fig. 3C).

\section{Discussion}

The TGFBI gene (also known as BIGH3) was first identified by Skonier et al in 1992 (14), and has been closely associated with inherited CDs. In the present study, mutations of TGFBI were identified in three unrelated Chinese families affected with LCDI, LCDIIIB and GCD2, respectively. Mutations were identified in all of the families, and there were perfect correlations between LCDI and R124C, between LCDIIIB and H626R, and between GCD2 and R124H.

LCD is generally divided into three subtypes, and classification has been assigned according to clinical and pathological findings. LCDI is an autosomal dominantly inherited corneal amyloidosis, which is characterized by a network of delicate linear opacities within the superficial corneal stroma. The disease usually begins in the first decade of life with symptoms of recurrent painful epithelial erosions. Lattice lines and diffuse opacification of the central cornea develop gradually subsequent to the formation of amyloid accumulations or erosions. Several studies from different ethnic groups have demonstrated an R124C mutation of the TGFBI gene as the most common cause of LCDI $(11,15,16)$. In the present study, the clinical features of the affected members of the family with LCDI were similar, however, Liu et al (17) reported that the clinical features of Chinese patients with the same R124C mutation were variable, even within the same family. Therefore, the mechanism underlying phenotypic variability with the same mutation and within the same family remains unclear and requires further investigation.

The LCDIIIB subtype is characterized by bilateral progressive visual impairment and has an intermediate age of onset, compared with LCDI and LCDIIIA. The LCDIIIB 
Table I. Primers for amplification of transforming growth factor $\beta 1$ regions.

Sequence $\left(5^{\prime}-3^{\prime}\right)$

\begin{tabular}{cll}
\cline { 2 - 3 } Exon & \multicolumn{1}{c}{ Forward } & \multicolumn{1}{c}{ Reverse } \\
\hline 1 & TC TCA CTT CCC TGG AG & GAC TAC CTG ACC TTC CGC AG \\
2 & GGT GGA CGT GCT GAT CAT CT & AGC CAG CGT GCA TAC AGC TT \\
3 & TTC ACC CAC CAT TCC TCT TC & GGT ACT CCT CTC TCC CAC CA \\
4 & ATC CCT CCT TCT GCT TTC TG & GCA GAC GGA GGT CAT CTC AC \\
5 & TTA AAC ACA GAG TCT GCA GC & TTC ATT ATG CAC CAA GGG CCA \\
6 & TGT TGA CTG CTC ATC CTT GC & CTC TTG GGA GGC AAT GTG TC \\
7 & CTT CAG GGA GCA CTC CAT C & AAT CTA GCT GCA CAA ATG AGG \\
8 & CTT GAC CTG AGT CTG TTT GGA & GGA TGG CAG AAG AGA TGG TG \\
9 & CCT GCT GAT GTG TGT CAT GC & CTG CCT CCA GGG ACA ATC TA \\
10 & TCA TTG CAG GAG CAC ATC TC & CCC AGG AGC ATG ATT TAG GA \\
11 & GAG GCC CCT CGT GGA AGT A & ACA TCC CAC TCC AGC ATG AC \\
12 & CTG TTG ACA GGT GAC ATT TTC & ATG TGC CAA CTG TTT GCT GCT \\
13 & GGG ATT AAC TCT ATC TCC TT & TGT GTA TAA TTC CAT CCT GG \\
14 & TCA GTA AAC ACT TGC TGA GTG AA & ACT GCC ACA TGG AGA AAA GGA C \\
15 & CCT CAG TCA CGG TTG TTA TG & CTC TAT GGC CCA AAC AGA GG \\
16 & TTG TCA TAA GCA GTT GCA GG & GCT TGC TTG GGG GTA AGG \\
17 & TCC TAG ACA GAC ATG GGG AGA & TGA GAG AAA TTG GCG GAG AG \\
\hline
\end{tabular}

A
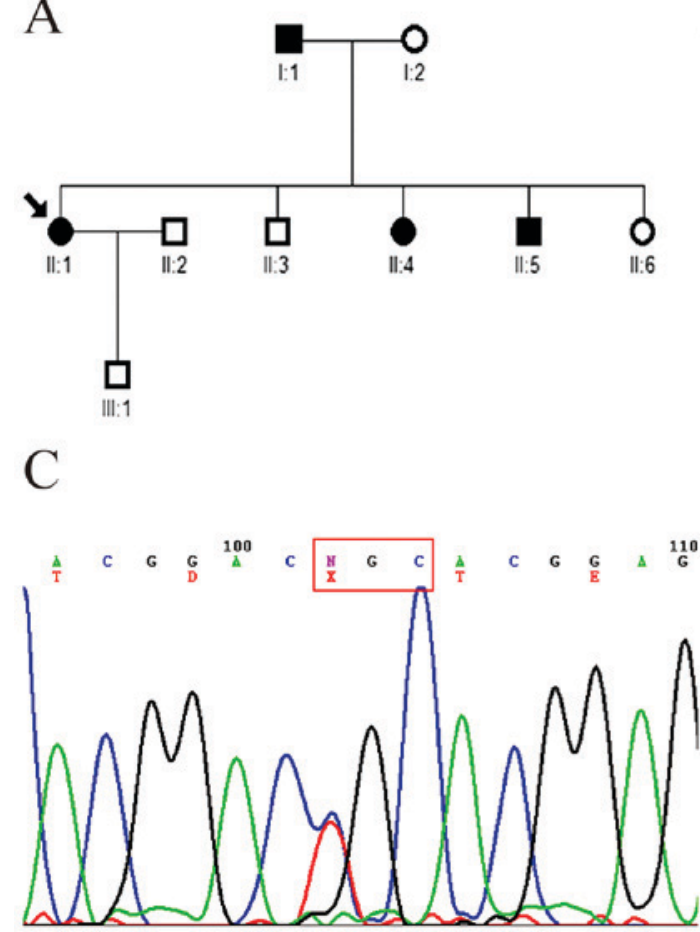

$\mathrm{B}$

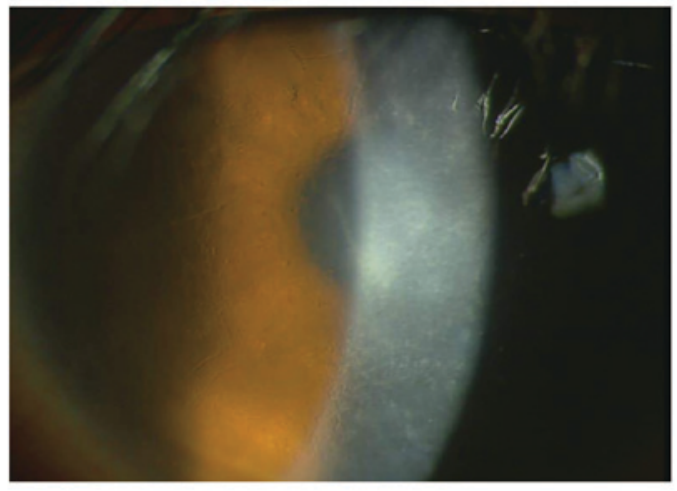

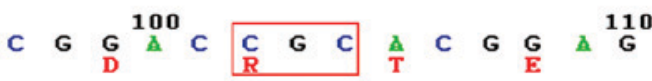

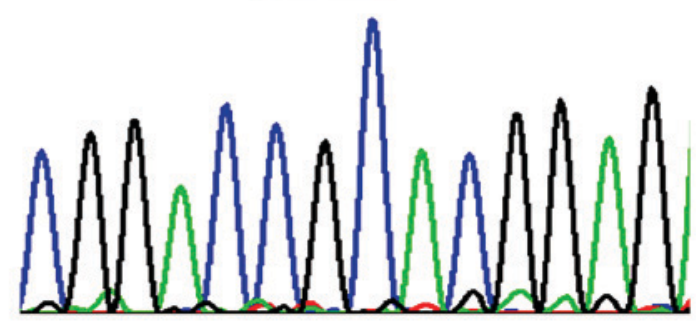

Figure 1. (A) Pedigree of family affected with LCDI. Open symbols indicate unaffected individuals; solid symbols indicate affected members. Arrows indicate the proband. (B) Corneal images of the proband show a network of linear opacities associated with other smaller opaque spots and refractile lattice lines. (C) Sequence around codon 124 within exon 4 in the patients with LCDI showing a nucleotide alteration at codon 124 (CGC $\rightarrow$ CTC), replacing an arginine with a cystine (R124C). LCSI, lattice corneal dystrophy type I.

subtype was first described in England (16), and was named by Chau et al (18). In 2010, Yang et al identified this mutation in a population in North China, which had clinical features of an intermediate subtype between LCDI and IIIA (19). In
2013, Wang et al first reported the TGFBI p.H626R mutation in a pedigree from South Chinese with LCDIIIB (20). All these findings suggested that TGFBI p.H626R may be a mutation hotspot across different populations in LCDIIIB. 


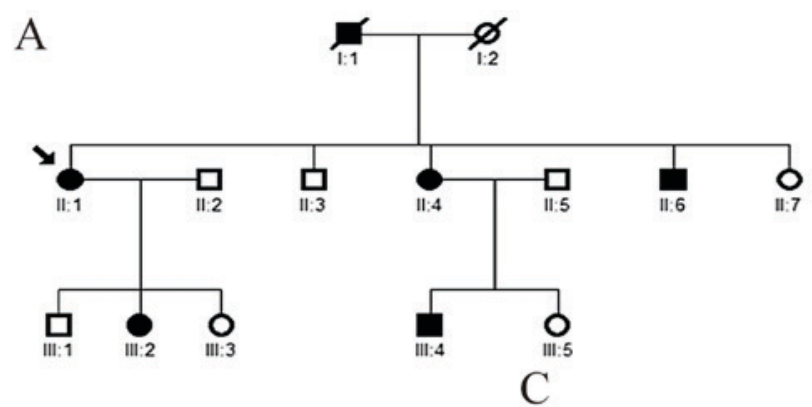

$\mathrm{B}$

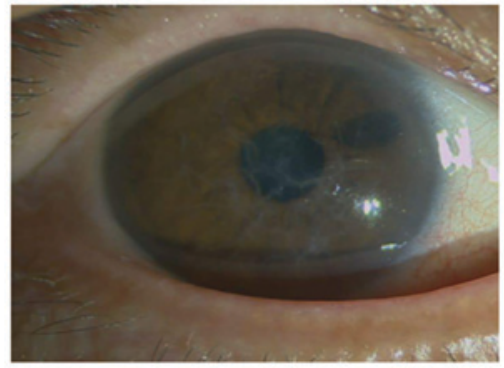

D

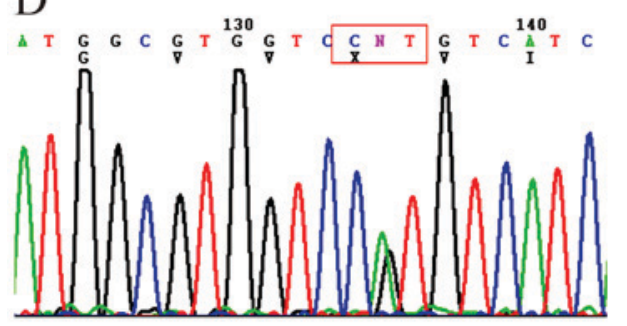

$\mathrm{C}$
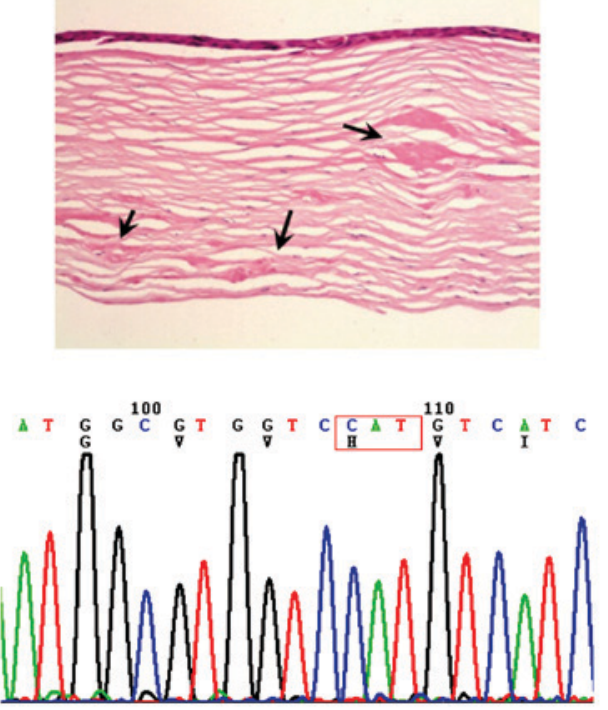

Figure 2. (A) Pedigree of family affected with the LCDIIIB. Open symbols indicate unaffected individuals; solid symbols indicate affected members. Arrows indicate the proband. (B) Corneal images of the proband show thick lattice lines extending to the periphery. A superficial scar is seen as a result of recurrent corneal erosions. (C) Histopathological appearance of the corneal buttons obtained from the proband. Arrows indicate amyloid deposits. (D) Sequence around codon 626 within exon 14 in the patients with LCDIIIB shows a nucleotide alteration at codon 626 (CAT $\rightarrow$ CGT), replacing a histidine with an arginine (H626R). LCDIIIB, lattice corneal dystrophy type IIIB.
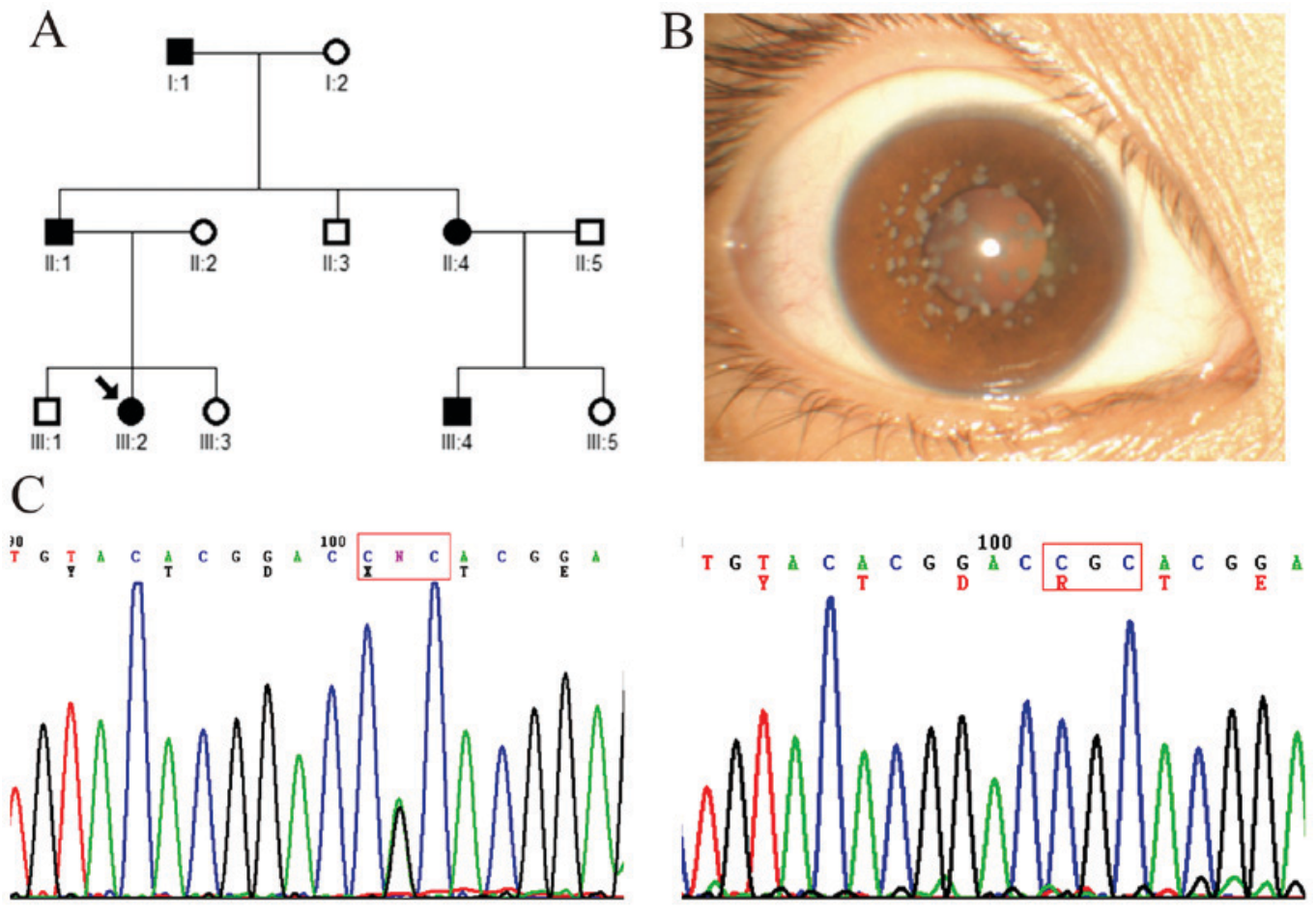

Figure 3. (A) Pedigree of family affected with the GCD2. Open symbols indicate unaffected individuals; solid symbols indicate affected members. Arrows indicate the proband. (B) Corneal images of the proband show scattered grayish small dot, annular and snow flake-like opacities in the superficial mid-stroma in the central cornea. (C) Sequence around codon 626 within exon 4 in the patients with GCD2 shows a nucleotide alteration at codon 124 (CGC $\rightarrow$ CAC), replacing an arginine with a histidine (R124H). GCD, granular corneal dystrophy type 2. 
Similar to the previous studies, the present study found the p.H626R mutation in the family affected by LCDIIIB.

The onset of GCD2, previously termed avellino dystrophy, often occurs in the second decade and demonstrates fewer opacities, compared with GCD1, exhibiting granular, branching deposits in a clear superficial mid-stroma, with lattice lines sometimes in deeper corneas (21). This disease has clinical and histologic features of granular and lattice dystrophy. To date, the molecular genetic techniques used for the majority of cases of GCD2 have revealed the R124H mutation in TGFBI, however, the phenotype of the affected individuals vary markedly in severity between families (22). Direct sequencing in the present study revealed that the c.371 G>A mutation of TGFBI resulting in Arg124His was responsible for GCD2 in the pedigree, which was consistent with the results of a previous study (21). These results further support the importance of $T G F B I$ in maintaining corneal transparency.

In conclusion, although no novel mutations were found in the three pedigrees with $\mathrm{CD}$ in the present study, the previously reported phenotype-genotype correlations were confirmed in all patients with $T G F B I$-linked CDs. As the mutation at $T G F B I$ can be readily, rapidly and cost-effectively detected via PCR sequencing or using PCR-RFLP, the identification of these mutations enables Chinese patients to benefit from the timely and accurate molecular diagnosis of CDs.

\section{Acknowledgements}

This study was supported by grants from the National Natural Science Foundation of China (grant no. 30940081) and the Xuzhou Science and Technology Foundation (grant no. XF10C050).

\section{References}

1. Weiss JS: Molecular genetics and the classification of the corneal dystrophies: What is next? Am J Ophthalmol 148: 477-478, 2009.

2. Weiss JS, Møller HU, Lisch W, Kinoshita S, Aldave AJ, Belin MW, Kivelä T, Busin M, Munier FL, Seitz B, et al: The IC3D classification of the corneal dystrophies. Cornea 27 (Suppl 2): S1-S83, 2008 (In English, Spanish).

3. Klintworth GK: Corneal dystrophies. Orphanet J Rare Dis 4: 7, 2009.

4. Klintworth GK: The molecular genetics of the corneal dystrophies-current status. Front Biosci 8: d687-d713, 2003.

5. Aldave AJ and Sonmez B: Elucidating the molecular genetic basis of the corneal dystrophies: Are we there yet? Arch Ophthalmol 125: 177-186, 2007.

6. Munier FL, Korvatska E, Djemaï A, Le Paslier D, Zografos L, Pescia $\mathrm{G}$ and Schorderet DF: Kerato-epithelin mutations in four 5q31-linked corneal dystrophies. Nat Genet 15: 247-251, 1997.
7. Cho KJ, Mok JW, Na KS, Rho CR, Byun YS, Hwang HS, Hwang KY and Joo CK: TGFBI gene mutations in a Korean population with corneal dystrophy. Mol Vis 18: 2012-2021, 2012.

8. Tian X, Fujiki K, Zhang Y, Murakami A, Li Q, Kanai A, Wang W, Hao Y and Ma Z: A novel variant lattice corneal dystrophy caused by association of mutation (V625D) in TGFBI gene. Am J Ophthalmol 144: 473-475, 2007.

9. Tian X, Fujiki K, Wang W, Murakami A, Xie P, Kanai A and Liu Z: Novel mutation (V505D) of the TGFBI gene found in a Chinese family with lattice corneal dystrophy, type I. Jpn J Ophthalmol 49: 84-88, 2005.

10. Yang QN, Zhao YW, Guo LH, Yan NH, Liu XY and Cai SP: Arg124Cys mutation of the TGFBI gene in a Chinese pedigree of Reis-Bücklers corneal dystrophy. Int J Ophthalmol 4: 235-238, 2011.

11. Patel DA, Chang SH, Harocopos GJ, Vora SC, Thang DH and Huang AJ: Granular and lattice deposits in corneal dystrophy caused by R124C mutation of TGFBIp. Cornea 29: 1215-1222, 2010.

12. Munier FL, Frueh BE, Othenin-Girard P, Uffer S, Cousin P, Wang MX, Héon E, Black GC, Blasi MA, Balestrazzi E, et al: BIGH3 mutation spectrum in corneal dystrophies. Invest Ophthalmol Vis Sci 43: 949-954, 2002.

13. Stix B, Leber M, Bingemer P, Gross C, Rüschoff J, Fändrich M, Schorderet DF, Vorwerk CK, Zacharias M, Roessner A and Röcken C: Hereditary lattice corneal dystrophy is associated with corneal amyloid deposits enclosing C-terminal fragments of keratoepithelin. Invest Ophthalmol Vis Sci 46: 1133-1139, 2005.

14. Skonier J, Neubauer M, Madisen L, Bennett K, Plowman GD and Purchio AF: cDNA cloning and sequence analysis of beta ig-h3, a novel gene induced in a human adenocarcinoma cell line after treatment with transforming growth factor-beta. DNA Cell Biol 11: 511-522, 1992

15. Kim HS, Yoon SK, Cho BJ, Kim EK and Joo CK: BIGH3 gene mutations and rapid detection in Korean patients with corneal dystrophy. Cornea 20: 844-849, 2001.

16. Stewart H, Black GC, Donnai D, Bonshek RE, McCarthy J, Morgan S, Dixon MJ and Ridgway AA: A mutation within exon 14 of the TGFBI (BIGH3) gene on chromosome 5q31 causes an asymmetric, late-onset form of lattice corneal dystrophy. Ophthalmology 106: 964-970, 1999.

17. Liu Z, Wang YQ, Gong QH and Xie LX: An R124C mutation in TGFBI caused lattice corneal dystrophy type I with a variable phenotype in three Chinese families. Mol Vis 14: 1234-1239, 2008.

18. Chau HM, Ha NT, Cung LX, Thanh TK, Fujiki K, Murakami A and Kanai A: H626R and R124C mutations of the TGFBI (BIGH3) gene caused lattice corneal dystrophy in Vietnamese people. Br J Ophthalmol 87: 686-689, 2003.

19. Yang J, Han X, Huang D, Yu L, Zhu Y, Tong Y, Zhu B, Li C, Weng $M$ and Ma X: Analysis of TGFBI gene mutations in Chinese patients with corneal dystrophies and review of the literature. Mol Vis 16: 1186-1193, 2010.

20. Wang D, Yao Y, Zhang M and Chen J: Genetic and phenotypic investigation of a Chinese pedigree with lattice corneal dystrophy IIIB subtype. Eye Sci 28: 144-147, 2013.

21. Gu Z, Zhao P, He G, Wan C, Ma G, Yu L, Zhang J, Feng G, He L and Gao L: An Arg124His mutation in TGFBI associated to Avellino corneal dystrophy in a Chinese pedigree. Mol Vis 17: 3200-3207, 2011.

22. Abazi Z, Magarasevic L, Grubisa I and Risovic D: Individual phenoty pic variances in a family with Avellino corneal dystrophy. BMC Ophthalmol 13: 30, 2013. 